\title{
The role of serum free light chain and M-protein measurements in determining recurrent disease in multiple myeloma after stem cell transplantation
}

\begin{abstract}
The detection of reoccurrence of multiple myeloma (MM) after stem cell transplant (SCT) is usually carried out by the use of serum and urine protein electrophoresis and immunofixation. We retrospectively studied the utility of serum free light chain assay (sFLC) in a population of post-SCT MM patients and compared it to traditional methods with serum protein electrophroesis (SPEP). We compared the value of sFLC vs SPEP for predicting reoccurrence and for earlier detection. We analyzed consecutive $24 \mathrm{MM}$ patients diagnosed between 2004 and 2009, who underwent SCT with concomitant follow-up with sFLC and M-protein from 2004 to 2012. An elevated M-protein was indicated by a level $\geq 0.2 \mathrm{~g} / \mathrm{dL}$. An abnormal sFLC was indicated by a $\kappa: \lambda$ ratio $>1.65$ or $<0.26$ accompanied by an increase of $>10 \mathrm{mg} / \mathrm{dL}$ of $\mathrm{sFLC}$ from the baseline. Of the 24cases, 22 had intact immunoglobulin MM (16IgG, kappa; $2 \operatorname{IgG}$ lambda; $2 \operatorname{IgA}$ lambda; $2 \operatorname{IgA}$ kappa) and 2cases had light chain MM. All 24cases (100\%) achieved a complete response or partial response to SCT as defined by the International Myeloma Working Group (IMWG). After SCT, 4(16.7\%) MM patients remained in remission. $12(50.0 \%)$ patients had a recurrence of MM with measurable increases in both sFLC and M-spike, 5(20.8\%) with increase in sFLC only and 3(12.5\%) with increase in $\mathrm{M}$-spike alone. Among the 20 patients with reoccurrence after SCT, 35\% had earlier detection of sFLC than M-spike, $10 \%$ had M-spike detected before sFLC, $15 \%$ had concurrent elevated sFLC and M-spike, 25\% had elevated sFLC only, and 15\% had elevated M-spike alone. The medium time period for detecting the elevated sFLC or $\mathrm{M}$-spike in patients undergoing reoccurrence after SCT was 3-6months vs. 6-9months (p-value: 0.0386; Mann-Whitney one-sided test). Our study concludes that sFLC should be used in conjunction with clinical and other traditional measures for the earliest detection of MM recurrence to ensure that the diagnosis is not missed by using SPEP assay alone.
\end{abstract}

Keywords: multiple myeloma, serum immunofixation electrophoresis, soft tissue plasmacytoma, mann-whitney test, partial response, specific immunoglobulin, asymptomatic myeloma, bone marrow, related organ or tissue impairment, clinical manifestation, plasma cell dyscrasias
Volume 2 Issue I - 2016

\section{Paul J Lee, Xiaoqin Zhu, Yuna Gong, Kristine Cornejo M, Lokinendi V Rao, Rabie Al- Turkmani M}

Department of Pathology, University of Massachusetts Medica School, USA

\author{
Correspondence: Paul J Lee, Department of Pathology, \\ University of Massachusetts Medical School, 365 Plantation \\ Street, Worcester, MA 01605, USA, Tel 5087936274 \\ Email Paul.Lee@umassmemorial.org
}

Received: January 05, 2016 | Published: January 19, 2016
Abbreviations: MM, multiple myeloma; PCD, plasma cell dyscrasias; sFLC, serum free light chains; IMWG, international myeloma working group; SIFE, serum immunofixation electrophoresis; SPEP, serum protein electrophoresis; CZE, capillary zone electrophoresis; IFE, immunofixation electrophoresis; PR, partial response; $\mathrm{CR}$, complete response

\section{Introduction}

Multiple myeloma (MM) is a type of plasma cell dyscrasias (PCD) that is characterized by an expansion of a monoclonal population of malignant plasma cells. Studies have shown that baseline measurements of serum free light chains (sFLC) have prognostic value in patients with newly diagnosed MM. ${ }^{1}$ The International Myeloma Working Group (IMWG) recommends the use of serum immunofixation electrophoresis (SIFE) and serum protein electrophoresis (SPEP) and measurement of sFLC among the tests to evaluate MM. ${ }^{2}$ Testing for the M-protein is considered integral for the diagnosis and monitoring progression of MM. Currently, sFLC assay as defined by the IMWG plays a secondary role in the diagnosis, only monitoring of reoccurrence and disease progression. Studies have demonstrated sFLC ratio can serve as a helpful test along with immunofixation electrophoresis and SPEP in determination of monoclonal gammopathy in the MM population. ${ }^{3}$ Early detection of advancement of MM is important as watchful waiting for reoccurrence of disease usually results in progression of asymptomatic disease to clinical manifestation. If disease progression is not detected early, treatment options become limited and the patient will have to suffer from high-dose chemotherapy resulting in treatment-related toxicity. ${ }^{4}$ New treatment options are available that can prevent end-organ damage of late-stage MM, but this prevention requires early detection of disease progression. ${ }^{5}$

The selection of a sub-population of MM patients in remission who have a higher likelihood to progress to symptomatic MM can improve their overall survival and morbidity. Several reports have demonstrated the usefulness of the SFLC assay for the detection of different PCDs. ${ }^{6}$ The traditional tests like SPEP have difficulty in detection of certain PCDs where low M protein consists of only sFLCs. The use of the $\kappa: \lambda$ ratios along with the absolute value of sFLC has rendered a more accurate diagnosis for the sub classification of PCDs (monoclonal gammopathy of undetermined significance [MGUS], MM, non-secretory MM, AL amyloidosis) along with determination of early disease progression. The role of sFLC assay 
in influencing clinical decisions is currently being evaluated. ${ }^{7}$ The question this study investigates is whether sFLC assay can play a role in monitoring the reoccurrence of MM population after bone marrow transplantation. A previous study by Uljon et al. ${ }^{8}$ determined that sFLC measurements did demonstrate obvious advantages in early detection of MM reoccurrence when compared with M-spike measurements. ${ }^{8}$ We analyzed serial data in 24 patients with treated MM to determine whether M-protein by SPEP or sFLC concentrations would be more sensitive in diagnosing recurrent disease after SCT.

\section{Methods and materials}

We analyzed $24 \mathrm{MM}$ patients diagnosed at our hospital between 2004 and 2009, who underwent SCT with concomitant follow-up with sFLC and M-protein from 2004 to 2012. These patients had to fulfill the International Myeloma Working Group (IMWG) criteria for symptomatic myeloma with M-protein $>3 \mathrm{~g} / \mathrm{dL}$ and/or bone marrow clonal cells $>10 \%$ with related organ or tissue impairment (ROTI) end-organ damage, which is typically manifested by increased calcium, renal insufficiency, anemia, or bone lesions (CRABs) attributed to the plasma cell proliferative process. Asymptomatic myeloma lacks evidence of ROTI. All patients had fulfilled the criteria for either asymptomatic or symptomatic multiple myeloma. All patients included in the study must have a) undergone SCT, b) achieved complete or partial response to therapy, and c) were routinely monitored by sFLC and SPEP.

The SCT with complete response to therapy is defined by IMWG. ${ }^{2}$ All 24patients included in the study underwent either autologous or allogeneic SCT. Remission of disease is defined as absence of bone marrow or blood findings of $\mathrm{MM}$ with disappearance of serum and urine M components on SPEP and immunofixation for 6weeks, along with absence of soft tissue plasmacytoma. Criteria used for evaluating disease response and progression in patients with multiple myeloma treated by high-dose therapy and hematopoietic SCT were those defined by the European Group for Blood and Marrow Transplant. ${ }^{9}$ After inducing remission of MM, patients were followed with interval sFLC and SPEP for monitoring of reoccurrence.

The serum free light chain assay was performed on the SPAPLUS analyzer (Freelite; The Binding Site, Birmingham, United Kingdom) using serum samples. ${ }^{10,11}$ It consists of two separate measurements, one to detect free kappa [reference range, $3.3-19.4 \mathrm{mg} / \mathrm{L}$ ] and the other to detect free lambda [reference range, $5.7-26.3 \mathrm{mg} / \mathrm{L}$ ] light chains. The clonality of the light chains is determined with calculation of $\kappa: \lambda$ ratio (reference range, $0.26-1.65$ ). If the FLC ratio is greater than 1.65 , kappa is considered to be the "involved" FLC and lambda the "uninvolved" FLC, and vice versa if the ratio is less than 0.26 . The absolute difference between the involved and uninvolved sFLC was defined as increase of $>10 \mathrm{mg} / \mathrm{dL}$ from the baseline.

The serum protein electrophoresis was performed using the CAPILLARYS 2 (Sebia, France) for the detection and identification of monoclonal proteins in serum samples. The CAPILLARYS 2 capillary zone electrophoresis (CZE)/immunosubtraction screened for an elevated M-protein $\geq 0.2 \mathrm{~g} / \mathrm{dL}$. The $\mathrm{M}$ spike was measured by quantifying the peak obtained by the SPEP, in combination with serum total protein measured on the AU680 instrument (Beckman Coulter, United States). Positive SPEP screens were reflexed to additional immunotyping by CZE on CAPILLARYS 2 or, in some cases with low MM levels, to immunofixation electrophoresis (IFE) on the HYDRASYS system (Sebia, France). The analysis looked at the total cohort and determined how many of the MM patients in remission had a reoccurrence. This was defined as at least one of the following criteria: reappearance of serum or urine M-protein by immunofixation or electrophoresis, development of $>5 \%$ plasma cells in the bone marrow, or appearance of any other sign of progression (eg new plasmacytoma, new lytic bone lesion). The average number of days in remission was measured following SCT. The reoccurrence rate in the cohort was calculated and the subtype of PCD was determined using SPEP/CZE/IFE and sFLC. The absolute increase of M protein was measured and the time interval to remission was analyzed. Three-month intervals were used as time points for evaluation of reoccurrence. Statistical analyses were performed using the Minitab software package. The earliest time periods when M-spike and sFLC were detected after reoccurrence were presented as medians, and the comparison among these two categorical variables was performed with Mann-Whitney test.

\section{Results}

Among the cohort of 24 patients, there were 16male patients and 8 female patients. The median age of the cohort is 63.4years (Table 1).The average total days of follow-up were 1263 days. Average days of follow-up after SCT were 693 days. Of the 24cases, 22 had intact immunoglobulin MM (16IgG, kappa; 2IgG lambda; 2IgA lambda; 2IgA kappa) and 2cases had light chain MM (Table 1). All 24cases $(100 \%)$ achieved a complete response (CR) or partial response (PR) to SCT as defined by the IMWG. After SCT, $20 / 24$ patients (83.3\%) had a recurrence of MM with a measurable increase in sFLC, M-spike or both. $4 / 24(16.7 \%)$ patients remained in remission with no abnormal sFLC or M-spike detected during the time period of followup. In the group of patients with a reoccurrence, the cohort was separated in five groups: 1) detection by earlier M-spike compared with sFLC 2) detection by earlier sFLC compared with M-spike 3 ) detection by sFLC only and 4) detection by M-spike alone 5) concurrent detection by sFLC and M-spike (Table 2). Among the 20 patients with recurrence, $5(25 \%)$ patients had increase in sFLC alone, $3(15 \%)$ patients had increase in M-spike only, 7(35\%) patients showed increased sFLC detected earlier than M-spike, 2(10\%) patients showed elevated M-spike before sFLC, and 3 (15\%) patients had concurrent increase in M-spike as well as sFLC. The earliest time period when abnormal sFLC was detected ranged from within 3 months to $15-18$ months after SCT in 17 patients with a median of 3-6months. Meanwhile, the earliest time period for M-spike detection ranged from within 3months to $21-24$ months after SCT in 15patients with a median of 6-9months. The median time period when the first abnormal sFLC appeared was 3-to-6-month earlier than that of the first abnormal M-spike (twosided: $\mathrm{p}=0.0773$; one-sided: $\mathrm{p}=0.0386$; Mann-Whitney test).

The cohort with the detection of reoccurrence by an earlier M-spike vs sFLC constituted 2/24(8.3\%) patients. Patient \#2 is a representative example of the cohort (Figure 1). sFLC lagged behind the M-spike by 130 days. The cohort with a measurable increase in sFLC prior to M-spike constituted 7/24 (29.2\%) patients. Patient \#4 is a representative example (Figure 2). The sFLC preceded the M-spike by 150 days. The cohort with increases in only sFLC constituted $5 / 24(20.8 \%)$ patients. Patient \#10 is a representative example (Figure $3)$. The cohort with detection of reoccurrence with M-spike alone constituted 3/24(12.5\%) patients. The last cohort with concurrent increase in both M-spike and sFLC constituted 3/24(12.5\%) patients. Patient \#19 is a representative example (Figure 4). 
Table I Summary of patient characteristics

\begin{tabular}{lllllll}
\hline Gender & \multicolumn{1}{c}{ Age } & *IIMM & & * LCMM & \\
\hline Male & 16 & $63.8 \pm 8.8$ & IgG Kappa & 14 & Kappa & 1 \\
Female & 8 & $62.8 \pm 10.5$ & IgG Lambda & 3 & Lambda & 1 \\
& & & IgA Kappa & 3 & & \\
& & & IgA Lambda & 2 & & \\
Total & 24 & $63.4 \pm 9.2$ & & 22 & & 2 \\
\hline
\end{tabular}

*IIMM-Intact Immunoglobulin Multiple Myeloma.

*LCMM-Light Chain Multiple Myeloma.

Table 2 Time after SCT to detection of abnormal sFLC, K/L or M-spike

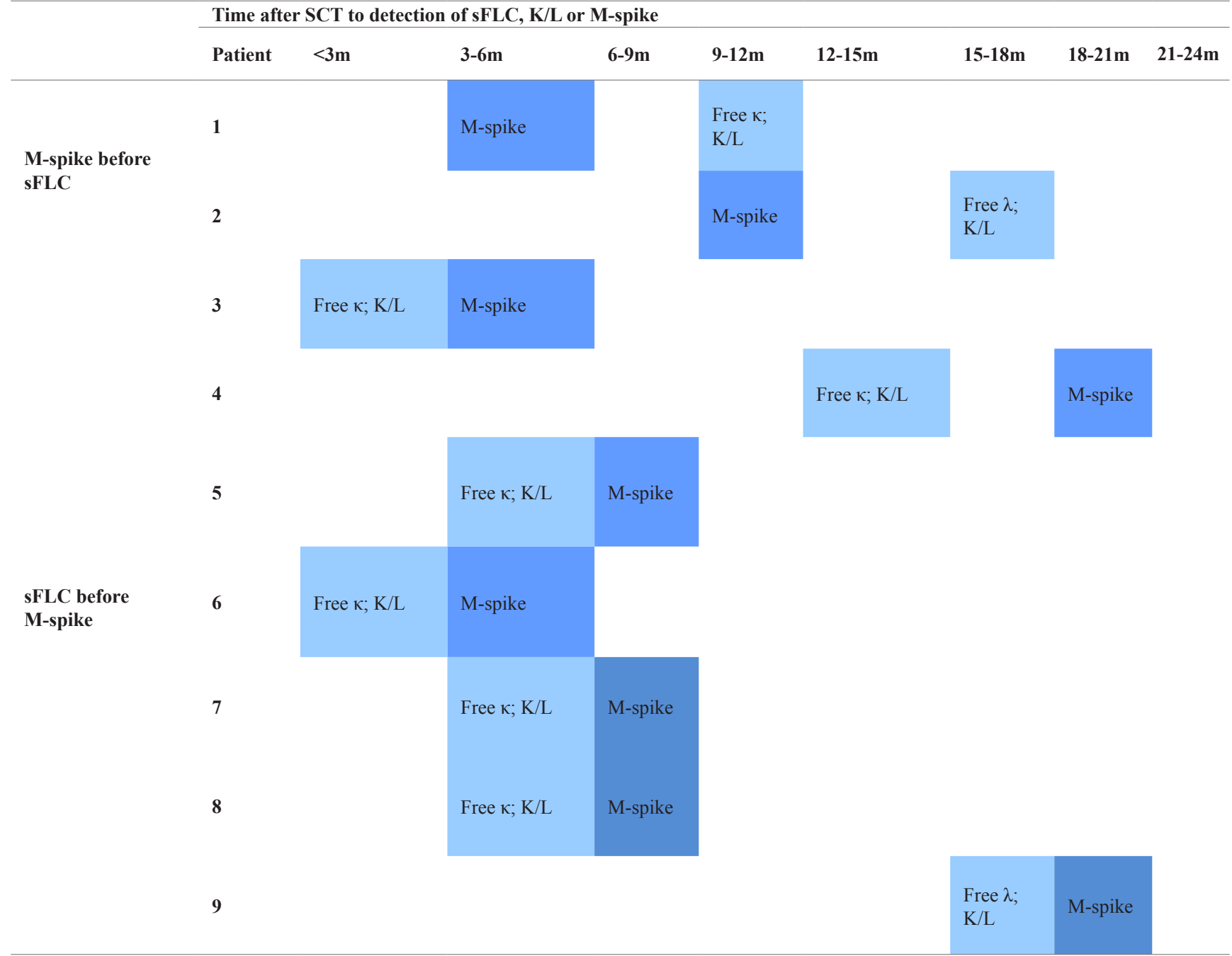


Time after SCT to detection of sFLC, K/L or M-spike

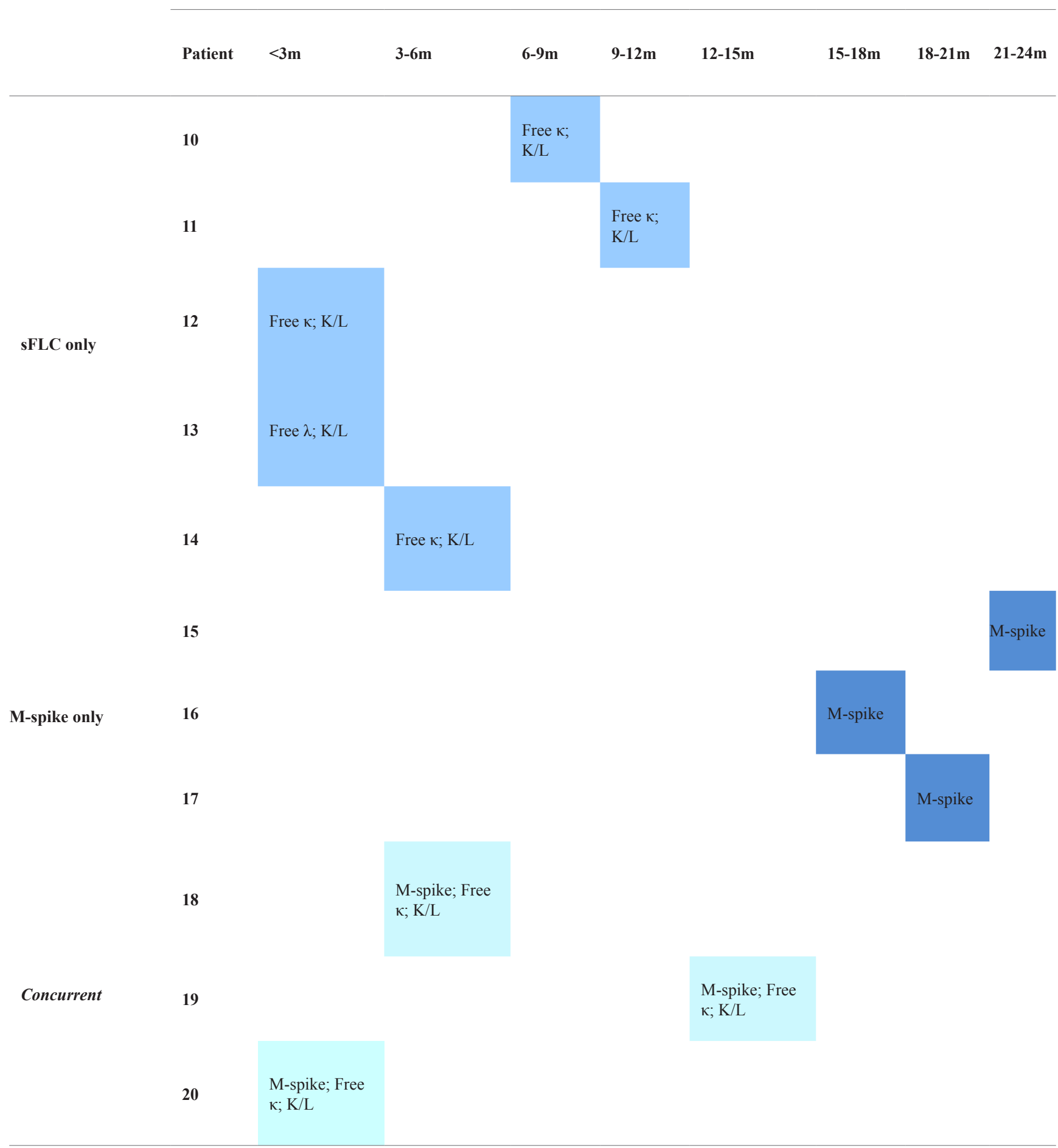




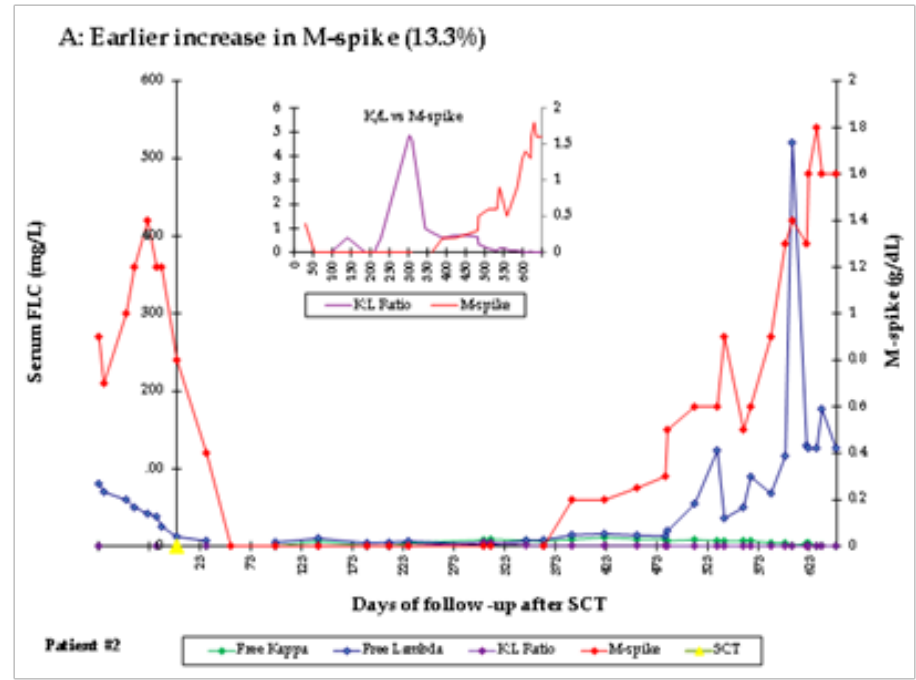

Figure I Patient \#2 with an early increase of M-spike compared with sFLC (I3.3\%)

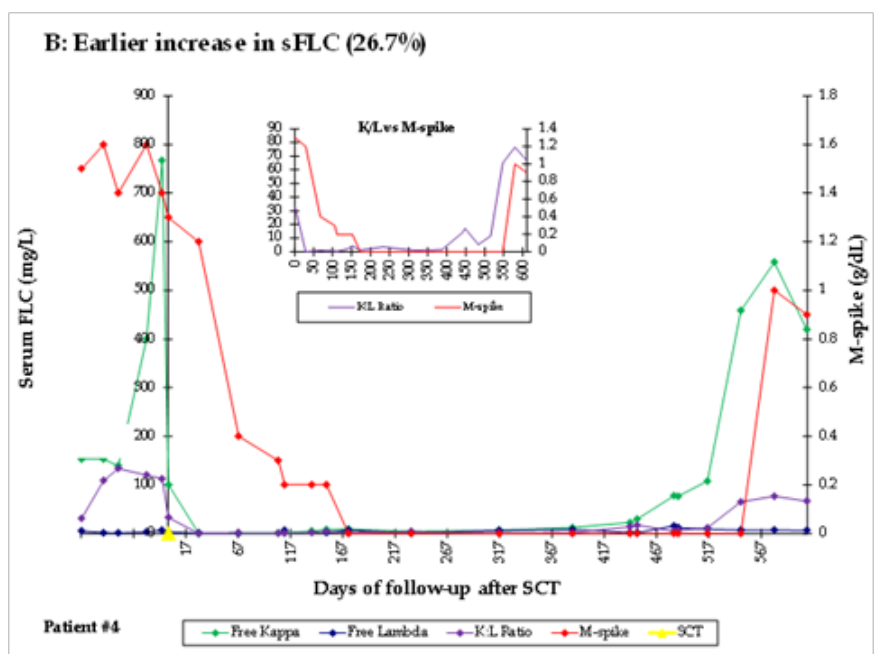

Figure 2 Patient \#4 demonstrating an early detection with sFLC vs M-spike.

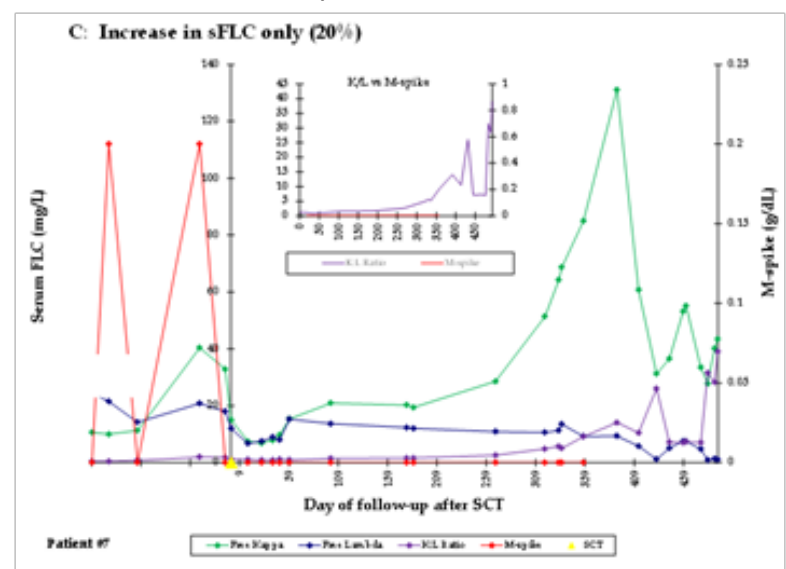

Figure 3 Patient \#I0 demonstrating detection of reoccurrence with only sFLC. 


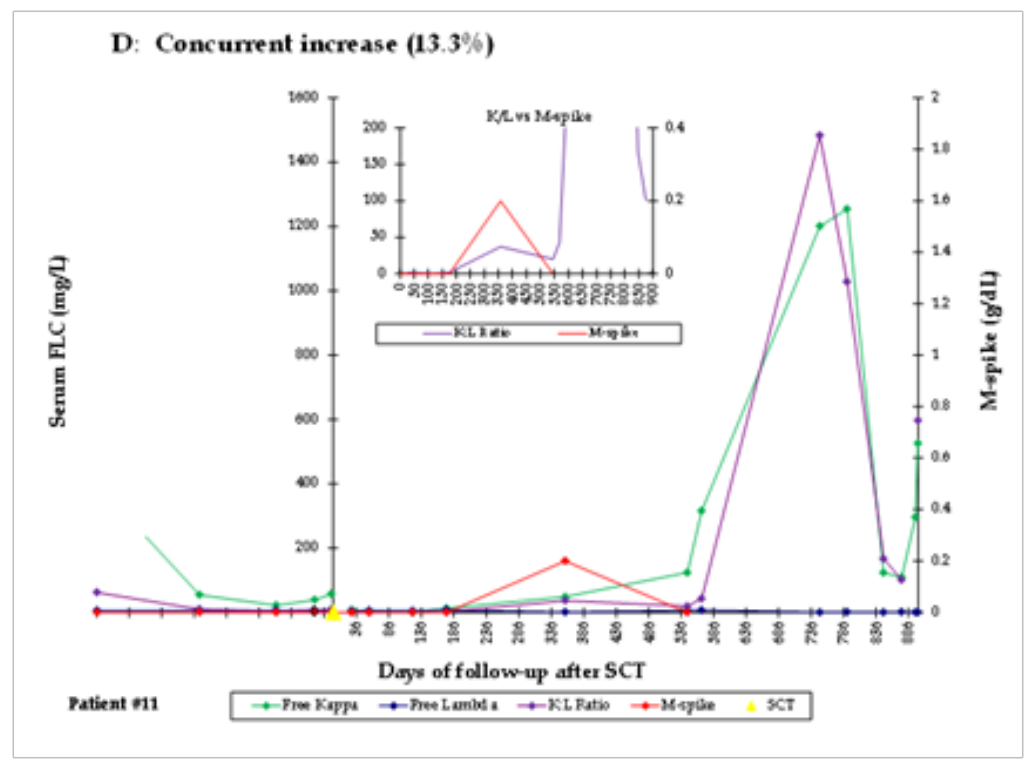

Figure 4 Patient \#I9 demonstrating concurrent detection by sFLC and M-spike.

\section{Discussion}

The analysis of 24patients with clinically diagnosed MM, who underwent SCT and were monitored for reoccurrence using M-spike and sFLC, did not demonstrate significant differences in the performance of these two parameters and their utility to monitor disease progression. The sFLC was detected in $70.8 \%$ of the cohort during the surveillance of 2years, which constituted $85.0 \%$ of the patient with reoccurrence. M-spike was detected in $62.5 \%$ of the cohort, which constituted $75.0 \%$ of the reoccurrence population. The majority of patients did relapse during the surveillance interval of 2years; however one method did not significantly demonstrate a superior performance for early detection of MM. The sFLC assay was more likely to detect the relapse of MM prior to SPEP. $29.2 \%$ of the patients had detectable sFLC abnormality prior to the presence M-spike, while the opposite was true in only $8.3 \%$ of the cohort. The sFLC could likely detect a reoccurrence after 3-6months, compared to 6-9months for M-spike. The shorter half-life of sFLC ( 2 to 6hours) vs complete immunoglobulin (21days) and the much lower detection limit of sFLC assay compared to SPEP could potentially explain the leading changes in sFLC measurements in MM patients with reoccurrence and the ability of sFLC assay to detect incremental changes in the sFLC at lower concentrations. Although the sFLC assay has been used in $\mathrm{MM}$ monitoring for years, there are certain $\mathrm{MM}$ conditions where sFLC is a helpful marker in detecting the disease. Examples include non-secretory MM as well as AAL amyloidosis proteins derived from only light chains, which are deposited in tissue with low concentration of the $\mathrm{M}$ chain and difficult to detect with $\mathrm{M}$-spike. In light chain MM, the M-protein consists only of free light chains, thus M-spike analysis would not always be useful and may miss a number of cases. Thus, the detection of a wider spectrum of disease can be better achieved by sFLC assay than by M-spike alone. There are limitations to the use of sFLC or M-spike alone for patient management that can lead to missed diagnosis of reoccurrence of MM.
Our study has shown that it is not recommended to use sFLC alone as a tool for monitoring MM patients in remission after SCT. There is a cohort of patients in our study that had an M-spike only without detectable sFLC, albeit a small portion $(12.5 \%)$ of reoccurrence population. The M-spike helps in surveillance of the specific immunoglobulin (IG) for comparison with the previously seen clonal plasma population. However, the use of M-spike alone for screen will not pick up many of the sFLC only cohort and there will be a delay in reoccurrence diagnosis by about 3 months. The two analytical techniques should be used in tandem to have the highest sensitivity for monitoring progression and reoccurrence of MM. Other pertinent findings include:

i. No relationship between reoccurrence and type of SCT (allogenic vs autologous) or the chemotherapy regimen used.

ii. The relationship of M-spike and sFLC was not affected by the specific isotype of M-protein in our cohort.

iii. No correlation was demonstrated between the subtype of MM and preferred method of early detection.

The variability in SPEPs and SFLC results may be interpreted within the context of individual patient MM tumor kinetics and differences in the analytical methods. Although the reoccurrence was more likely to be observed with sFLC with a lagging M-spike, concurrent and preceding $\mathrm{M}$-spike cases were observed. A cautious interpretation is recommended for sFLC and M-spike results when monitoring for $\mathrm{MM}$ disease progression after marrow ablation and SCT.

\section{Acknowledgements}

None.

\section{Conflict of interest}

The author declares no conflict of interest. 


\section{References}

1. Larsen JT KS, Dispenzieri A, Kyle RA, et al. Serum free light chain ratio as a biomarker for high-risk smoldering multiple myeloma. Leukemia. 2013;27(4):941-946.

2. Dispenzieri A, Kyle R, Merlini G, et al. International myeloma working group guidelines for serum-free light chain analysis in multiple myeloma and related disease. Leukemia. 2009;23(2):215-224.

3. Weiss BM AJ, Verma P, Howard RS, et al. A monoclonal gammopathy precedes multiple myeloma in most patients. Blood. 2009;113(22):54185422 .

4. Gertz MA. Immunoglobulin light chain amyloidosis: 2013 update on diagnosis, prognosis, and treatment. Am J Hematol. 2013;88(5):417-422.

5. Podar K TY, Hideshima T, Vallet S, et al. Emerging therapies for multiple myeloma. Expert Opin Emerg Drugs. 2009;14(1):99-127.

6. van Rhee F, Bolejack V, Hollmig K, et al. High serum-free light chain levels and their rapid reduction in response to therapy define an aggressive multiple myeloma subtype with poor prognosis. Blood. 2007;110(3):827832.
7. Phillips DP TD, Hawkins CA, Hickman PE. Utilisation of sFLC assays how well do we comply with guidelines? Int J Lab Hem. 2013;35(2):200 210 .

8. Uljon SN RP, Schur PH, Anderson KC, et al. Serial serum free light chain measurements do not detect changes in changes in disease status earlier than electrophoretic M-spike measurements in patients with intact immunoglobulin myeloma. Clin Chem Acta. 2011;412(7-8):562-568.

9. BladÉ J SD, Reece D, Apperley J, et al. Criteria for evaluating disease response and progression in patients with multiple myeloma treated by high-dose therapy and haematopietic stem cell transplantation. $\mathrm{Br} J$ Haematol. 1998;102(5):1115-1123.

10. Mead GP, Carr-Smith HD, Drayson MT, et al. Serum free light chains for monitoring mulitple myeloma. Br J Haematol. 2004;125(3):348-354.

11. Bradwell AR. Serum free light chain measurements move to center stage. Clin Chem. 2005;51(5):805-807. 